\title{
Disparity in outcomes of melanoma adjuvant immunotherapy by demographic profile
}

\author{
Alexandra Ikeguchi*,1, Michael Machiorlatti² \& Sara K Vesely ${ }^{\text {iD }}$ \\ ${ }^{1}$ Hematology-Oncology Section, Department of Medicine, College of Medicine University of Oklahoma Health Sciences Center, \\ Oklahoma City, OK, USA \\ ${ }^{2}$ Department of Biostatistics \& Epidemiology, Hudson College of Public Health University of Oklahoma Health Sciences Center, \\ Oklahoma City, OK, USA \\ *Author for correspondence: Alexandra-lkeguchi@ouhsc.edu
}

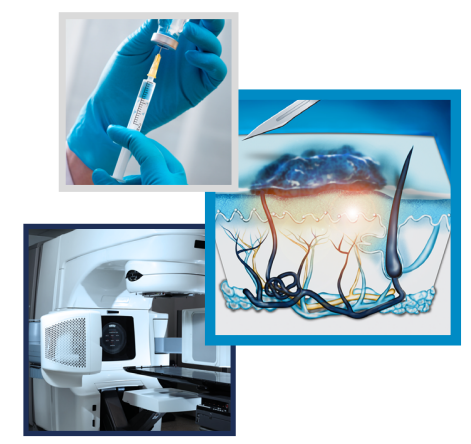

Background: Randomized comparisons have demonstrated survival benefit of adjuvant immunotherapy in node-positive melanoma patients but have limited power to determine if this benefit persists across various demographic factors. Materials $\&$ methods: We assessed the impact of demographic factors on the survival benefit of adjuvant immunotherapy in a database of 38,189 node-positive melanoma patients using the Kaplan-Meier method and Cox proportional hazards models. Results: All assessed demographic factors other than race significantly impacted survival of node-positive melanoma patients in univariate analysis. In multivariable analysis, only the age group interacted with immunotherapy. Conclusion: Analysis of this large database of unselected node-positive melanoma patients demonstrated a positive survival benefit of immunotherapy across all demographic factors assessed and the impact was greater for patients 65 years of age and older.

\section{Graphical abstract:}

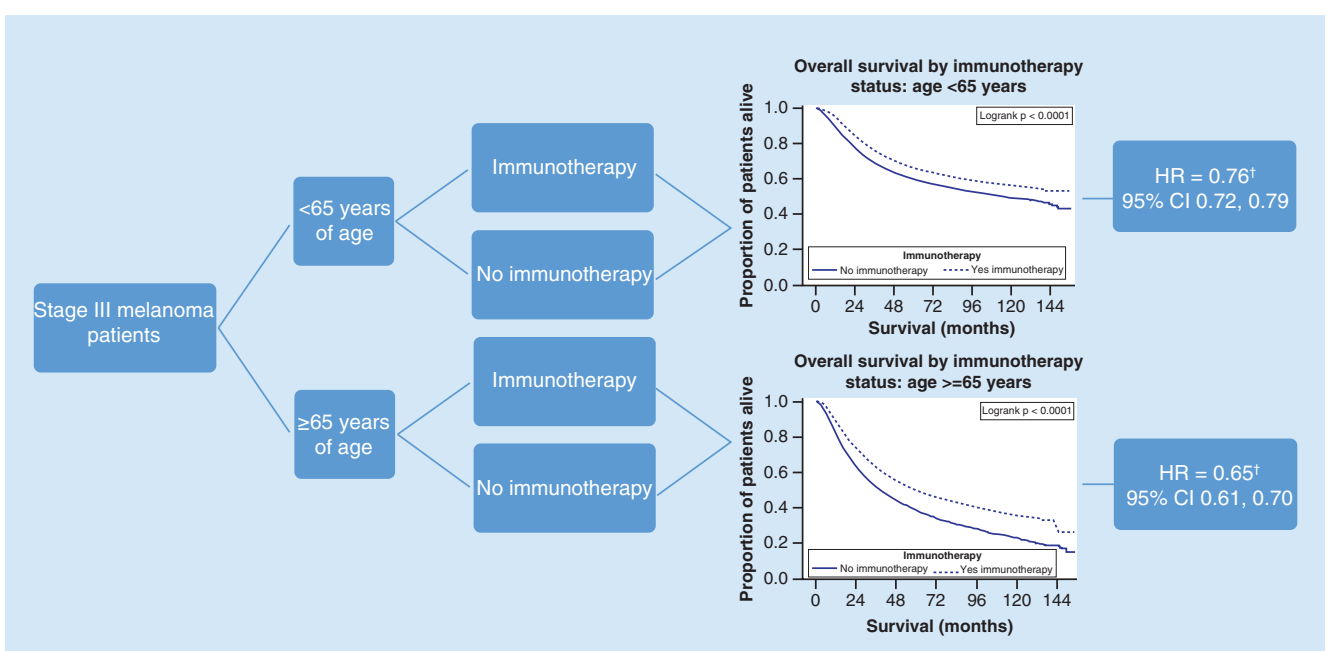

Stage III melanoma patients who received immunotherapy were at a decreased hazard for death. The protective effect from immunotherapy was greater for those $\geq 65$ years of age compared to those $<65$ years of age.

${ }^{\dagger}$ Hazard ratio adjusted for sex, race, insurance status, number of nodes and Charlson-Deyo score.

HR: Hazard ratio.

First draft submitted: 3 February 2020; Accepted for publication: 1 May 2020; Published online: 16 July 2020

Keywords: demographic factor • immunotherapy • melanoma • node-positive $\bullet$ survival

Malignant melanoma is a diagnosis that has dramatically increased in incidence over the past 40 years to become the fifth most common malignancy in the USA. In 2018, there were an estimated 91,270 new cases and 9320 
deaths $[1,2]$. However, cutaneous melanoma remains a relatively rare diagnosis in darker-skinned individuals and other demographic shifts in the profile of melanoma patients such as age at presentation have been noted as well. Treatment options for patients with advanced cutaneous melanoma have included surgical excision, immunotherapy, targeted therapy and radiation therapy to symptomatic sites of bulk disease, but prognosis for those with stage III-IV disease was uniformly poor until quite recently, with a 5 -year survival rate of approximately $20 \%$ during the time period 2007-2013 [1,3]. Immunotherapy and targeted therapy have been associated with highly significant improvements in survival [4-7]. However, individual trials have insufficient power to determine if these therapies are beneficial to all demographic subsets of patients, especially those subsets which may be underrepresented in clinical trials such as racial minorities and the elderly.

With respect to racial minorities, there are theoretical reasons to consider that the benefit of immunotherapy would not be as significant. African-Americans and Asians present most commonly with the acral lentiginous type of melanoma [8,9] and this subtype has a lower overall mutational burden [10], which could decrease responsiveness to immunotherapy. At the same time, Surveillance, Epidemiology and End Results Program (SEER) data indicate that African-Americans are more likely to present with later stages of melanoma [8]. The SEER database, therefore, has been insufficient to attribute the less favorable outcomes in racial minorities to either demographic factors affecting access to care or underlying differences in biology. Likewise, the interaction of melanoma survival and age appears to be complex [11], with favorable factors such as lower incidence of sentinel lymph node positivity offset by higher negative survival impact when a positive sentinel node is detected.

We proposed to review the National Cancer Database (NCDB) melanoma cases from 2004 to 2015, the most recent interval for which data had been entered at the time of our application, to evaluate the impact of immunotherapy in a much larger and unselected group of patients; we thereby sought to include those patients whose demographic profile is underrepresented in prior analyses. We sought to determine if the benefit of immunotherapy is impacted by these more diverse factors. This large database provides more robust information than SEER on each included individual such as payer status, distance to healthcare facility and comorbidity score along with basic demographic factors such as age and sex, which are known to have significant effect on overall survival of melanoma patients. Because many new therapies became widely available primarily for patients with unresectable stages III and IV cutaneous melanoma during this time period, we decided to narrow our focus to the results of adjuvant therapy for stage III. A small number of such patients could have received treatment with an immune checkpoint inhibitor (ICI) in the adjuvant setting on clinical trial, but IFN- $\alpha$ was the only approved regimen for virtually this entire time period. Thus, we anticipated our focus to be on the relative effectiveness of an immunotherapy strategy between different demographic groups rather than on the overall effectiveness of a specific regimen.

\section{Materials \& methods}

Data source

The NCDB is a joint sponsored project of the Commission on Cancer (CoC) of the American College of Surgeons and the American Cancer Society. The NCDB sources data from over 1500 hospital registries from CoC-accredited hospitals. The NCDB cites that more than $70 \%$ of all newly diagnosed malignancies in the USA are captured in the database. In accordance with the Health Insurance Portability and Accountability Act (HIPAA), the data in the NCDB is de-identified of specific patient and hospital information and can be used for public analysis [12].

\section{Study design}

Data was obtained from a melanoma participant user file from the NCDB to explore the outcome disparities of melanoma patients by demographic, health and treatment profile. The data contained patients between 2004 and 2015 with a diagnosis of melanoma. The variables of interest were survival time in months (outcome) with covariates of age, sex, race, insurance status, number of positive nodes, Charlson-Deyo score and adjuvant immunotherapy. Overall survival (OS) was defined as the number of months between date of diagnosis and dates of death (if known) or last follow-up (last known alive date). The University of Oklahoma Health Sciences Center Institutional Review Board determined this study was exempt since the data was de-identified. The Charlson-Deyo score is a weighted score derived from the sum of the scores for each of the comorbid conditions listed in the Charlson Comorbidity Score Mapping Table $[13,14]$. Some of the variables included are age, myocardial infarction, dementia, liver disease and a host of other conditions that indicate a worsening health prognosis of the patient. By incorporating the variable into the model, the analysis can be adjusted for competing conditions and allow for comparison of patients with similar health backgrounds. A detailed flow diagram shows exclusion criteria with number excluded 


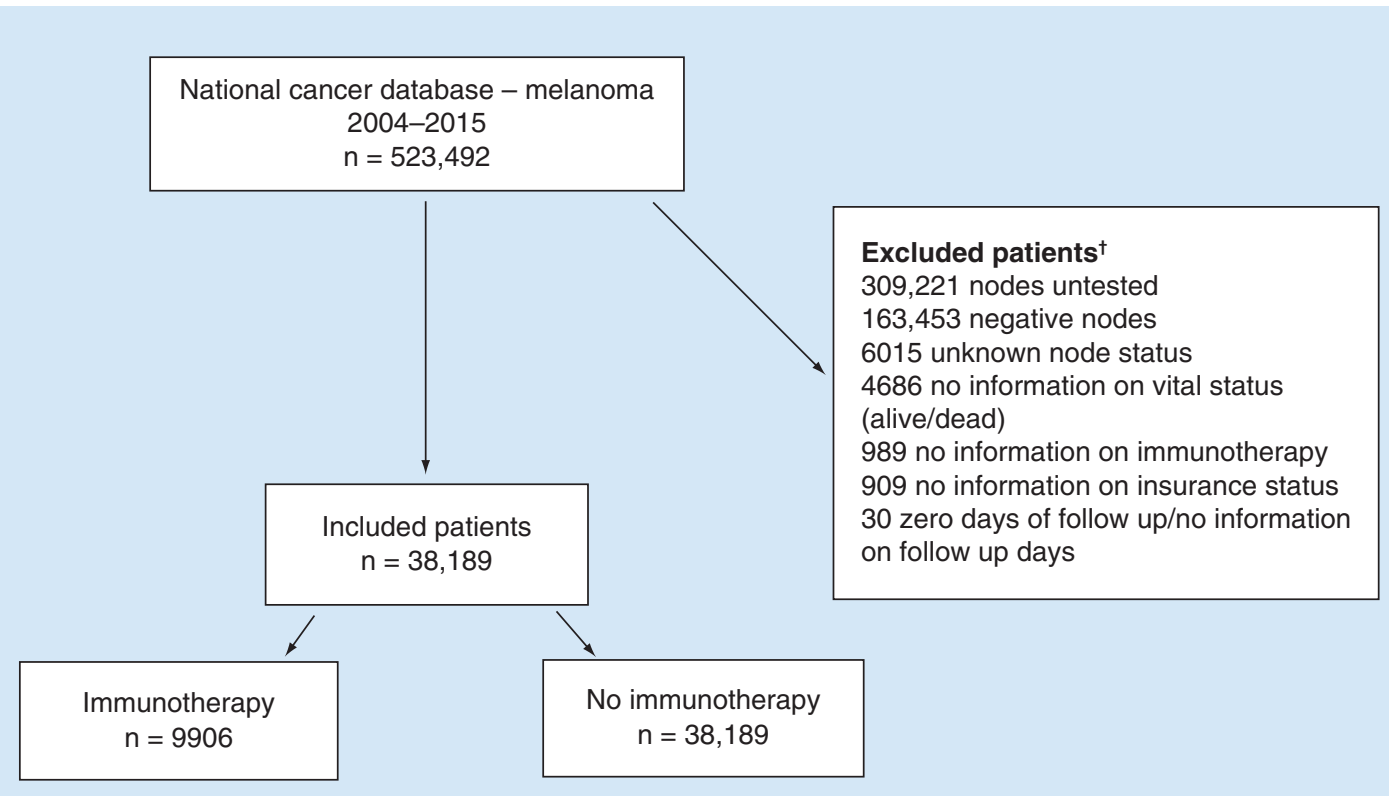

Figure 1. Flow diagram for inclusion of stage III melanoma patients.

$\dagger$ Patients excluded in a hierarchical fashion.

(Figure 1). First, only stage III patients were included for analysis; therefore, patients who had negative nodes, nodes that were not tested or had incomplete information about nodes were excluded. Our primary exposure of interest was immunotherapy and our primary outcome of interest was time to death, therefore patients missing data on immunotherapy treatment or on time to death (including patients with 0 days of follow-up) were excluded. We also excluded patients with unknown insurance status. The total number of patients analyzed was 38,189 patients.

\section{Statistical analysis}

SAS 9.4 was used to perform all analysis. Patient's demographic and clinicopathologic characteristics were reported categorically using frequency statistics ( $\mathrm{n}$ and \%) and compared by chi-square test. For age, the median and range were reported. Median OS with 95\% CIs were calculated using the Kaplan-Meier method and differences in median OS were compared between covariates using the log-rank test. Cox proportional hazards models were calculated to estimate the hazard ratio for immunotherapy while controlling for all other covariates regardless of their p-value. Since immunotherapy was the exposure of interest, we evaluated all two-way interactions between immunotherapy and all other covariates using an alpha of 0.001 to take into account multiple testing. All covariates remained in the final model and if interactions were identified then the data was stratified by one of the covariates involved in the interaction. Within strata, two-way interactions were assessed with an alpha of 0.001 also taking into account clinical relevance (i.e., focusing on interactions that were different by a clinically significant amount or were in opposite directions). Kaplan-Meier plots evaluating age group, immunotherapy and both age group and immunotherapy were created. An alpha of 0.05 was used unless otherwise noted.

\section{Results}

\section{Patient characteristics}

The demographic and clinical characteristics of the 31,189 patients are presented in Table 1 . The median age at diagnosis was 59 years with a range of $18-90$. Roughly $90 \%$ of patients were Caucasian with about $63 \%$ males. The most common types of insurance were private (54.1\%) and Medicare (34.9); only 4.6\% of patients were uninsured. Most patients (84.2\%) had a Charlson-Deyo score of zero. About 26\% ( $\mathrm{n}=9906)$ of patients received immunotherapy. Characteristics were compared among patients who did and did not receive immunotherapy (Table 1). Immunotherapy was received more often among patients who were $<65$ years of age, female, had private insurance, had more nodal involvement or had a Charlson-Deyo score of 0. 


\begin{tabular}{|c|c|c|c|c|c|}
\hline Variable & Class & All patients $(n=38,189)$ & $\begin{array}{l}\text { Immunotherapy } \\
(\mathrm{n}=9906)\end{array}$ & $\begin{array}{l}\text { No immunotherapy } \\
(n=28,283)\end{array}$ & p-value \\
\hline \multirow[t]{2}{*}{ Age } & $<65$ years & $24,049(63.0)$ & 8277 (83.6) & $15,772(55.8)$ & $<0.0001$ \\
\hline & $\geq 65$ years & $14,140(37.0)$ & 1629 (16.4) & $12,511(44.2)$ & \\
\hline Race & Other & $3598(9.4)$ & $957(9.7)$ & $2641(9.3)$ & 0.3435 \\
\hline Sex & Female & $14,160(37.1)$ & 3954 (39.9) & $10,206(36.1)$ & \\
\hline \multirow[t]{3}{*}{ Insurance status } & Not insured & $1761(4.6)$ & $505(5.1)$ & $1256(4.4)$ & $<0.0001$ \\
\hline & Private insurance & $20,657(54.1)$ & $7033(71.0)$ & $13,624(48.2)$ & \\
\hline & $\begin{array}{l}\text { Medicaid/other } \\
\text { government }\end{array}$ & $2452(6.4)$ & $758(7.7)$ & $1694(6.0)$ & \\
\hline Positive nodes, $\mathrm{n}$ & $\begin{array}{l}\text { Four or more nodes or } \\
\text { positive nodes } \\
\text { documented but number } \\
\text { unspecified }\end{array}$ & $5362(14.0)$ & $1465(14.8)$ & $3897(13.8)$ & \\
\hline \multirow[t]{3}{*}{ Charlson-Deyo score } & 0 & $32,144(84.2)$ & $8754(88.4)$ & $23,390(82.7)$ & $<0.0001$ \\
\hline & 1 & $4947(13.0)$ & $1014(10.2)$ & $3933(13.9)$ & \\
\hline & 2 or 3 & $1098(2.9)$ & $138(1.4)$ & $960(3.4)$ & \\
\hline \multirow[t]{2}{*}{ Vital status } & Dead & $16,872(44.2)$ & $3578(36.1)$ & $13,294(47.0)$ & $<0.0001$ \\
\hline & Alive & $21,317(55.8)$ & $6328(63.9)$ & $14,989(53.0)$ & \\
\hline
\end{tabular}

\section{Univariate analysis of overall survival}

Of the 38,189 patients, at last follow-up 44.2\% ( $n=16,872)$ had died. Using Kaplan-Meier survival analyses, unadjusted median OS stratified by various covariates were calculated (Table 2). Patients undergoing immunotherapy had a significantly higher median OS compared with those who did not (140.4 vs 60.2 months; $\mathrm{p}<0.0001$; Figure 2). Patients who were under 65 years of age had a far higher median OS compared with older patients (136.3 vs 39.2 months; $\mathrm{p}<0.0001$; Figure 3 ). Also, patients who were female, Caucasian race, had a low Charlson-Deyo score, had private insurance or had less cancerous nodes had higher median OS.

\section{Multivariable analysis of overall survival}

To determine the relationship between immunotherapy and survival analysis, we carried out a multivariable Cox proportional hazards analysis to control for competing risk factors as well as comorbid conditions. Among all patients, only the interaction between immunotherapy and age had p-value $<0.001$. We therefore stratified by age creating two models presented in Table 3 and created a Kaplan-Meier plot to show the age group and immunotherapy survival curves (Figure 4). Although immunotherapy significantly decreases risk of death for all patients, the magnitude of the difference is greater in patients 65 years and older. For patients less than 65 years, those who received immunotherapy had a higher median survival as compared with those who did not (median OS was not achieved for those received immunotherapy (at 155 months $53 \%$ were still alive) vs median OS in months of 114.6 ; $95 \%$ CI: $107.5-127.5)$. For patients who were 65 years or older, those who received immunotherapy had a higher median survival compared with those who did not (median OS in months 58.8; 95\% CI: 52.5-66.3 vs 37.5; 95\% CI: 36.2-38.5). Within the age group strata, there were no further interactions that met our criteria. Results from the multivariable Cox proportional hazards model show that for patients less than 65 years of age, after controlling for sex, race, insurance status, number of positive nodes and Charlson-Deyo score, immunotherapy decreased the risk of death by $24 \%$ (hazard ratio [HR]: 0.76 ; 95\% CI: $0.72-0.79$ ). After controlling for other variables in the model, females had a decreased risk of death (HR: 0.68; 95\% CI: 0.65-0.71) and race did not significantly impact risk of death. Compared with private insurance, having other forms of insurance increased your risk of death after controlling for other variables in the model (other insurance comparisons shown in Supplementary 


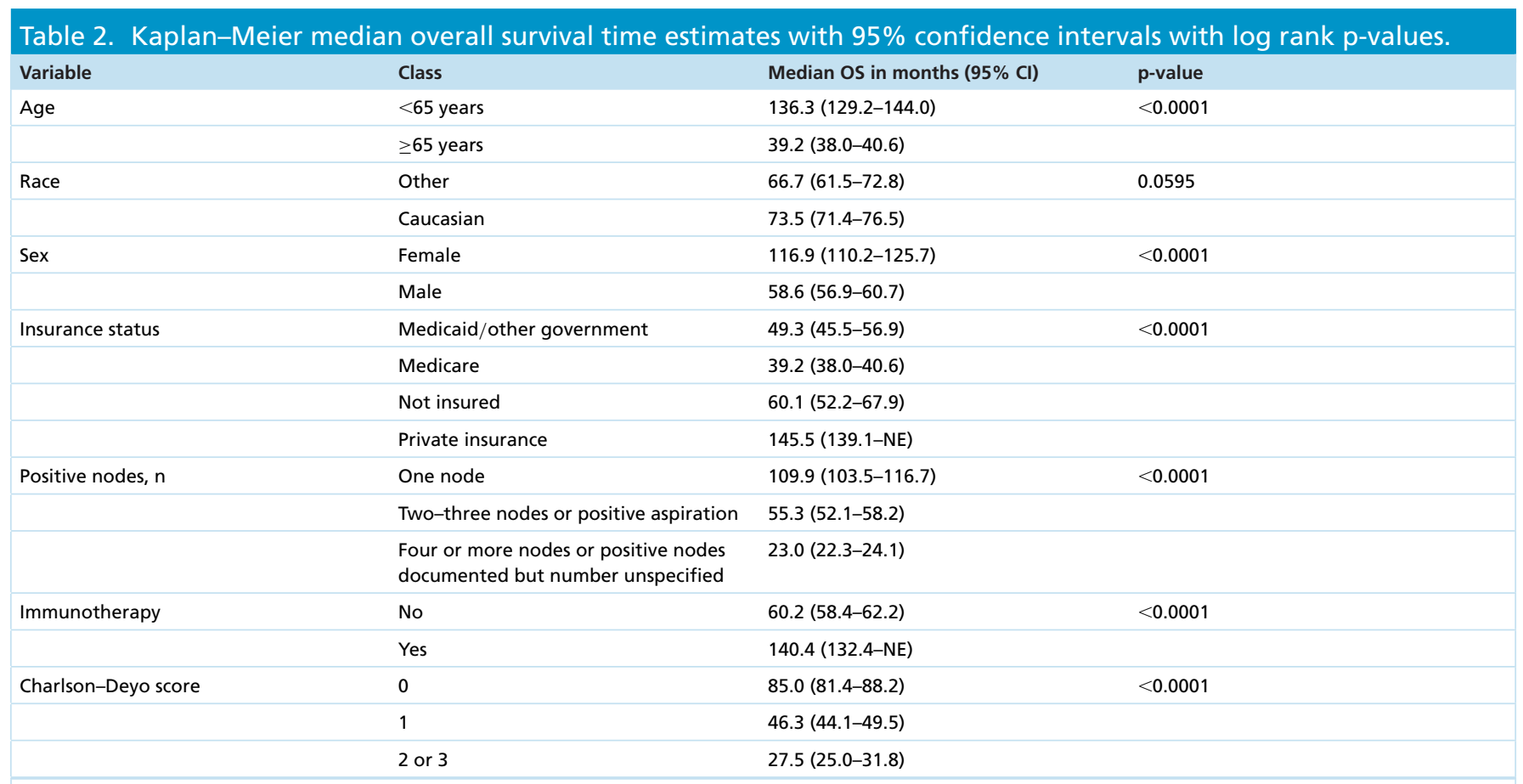

NE: No estimate; NR: Not reached; OS: Overall survival.

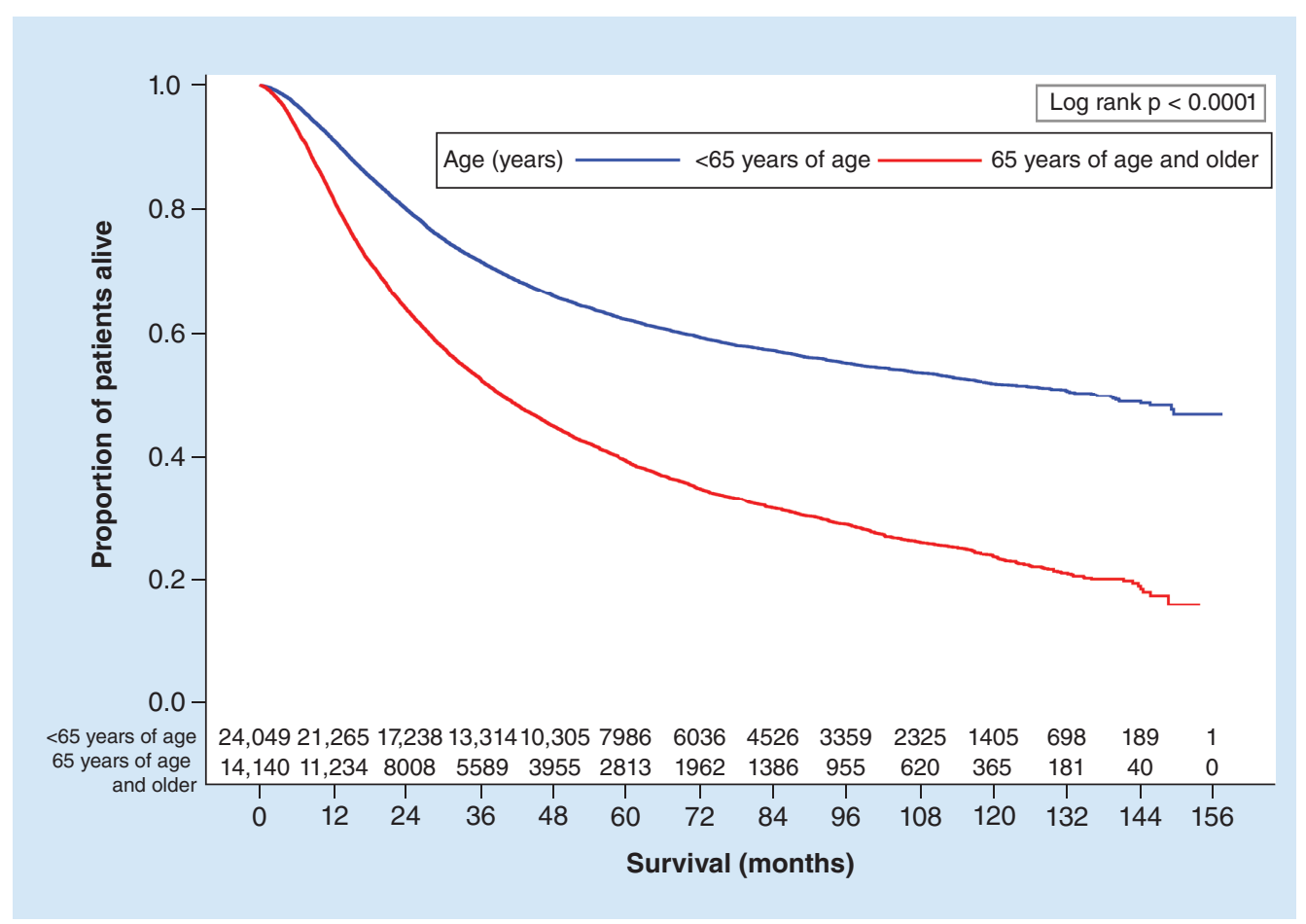

Figure 2. Kaplan-Meier overall survival plot with number at risk by age group ( $<65$ vs $\geq 65$ years).

Table 1). Increased risk of death in the model was shown for patients with more than one cancerous node and patients with a Charlson-Deyo score greater than zero. For patients 65 years or older, immunotherapy decreased the risk of death by 35\% (HR: 0.65; 95\% CI: 0.61-0.70) after controlling for sex, race, insurance status, number of positive nodes and Charlson-Deyo score. Based on the multivariable analysis, females had a decreased risk of 


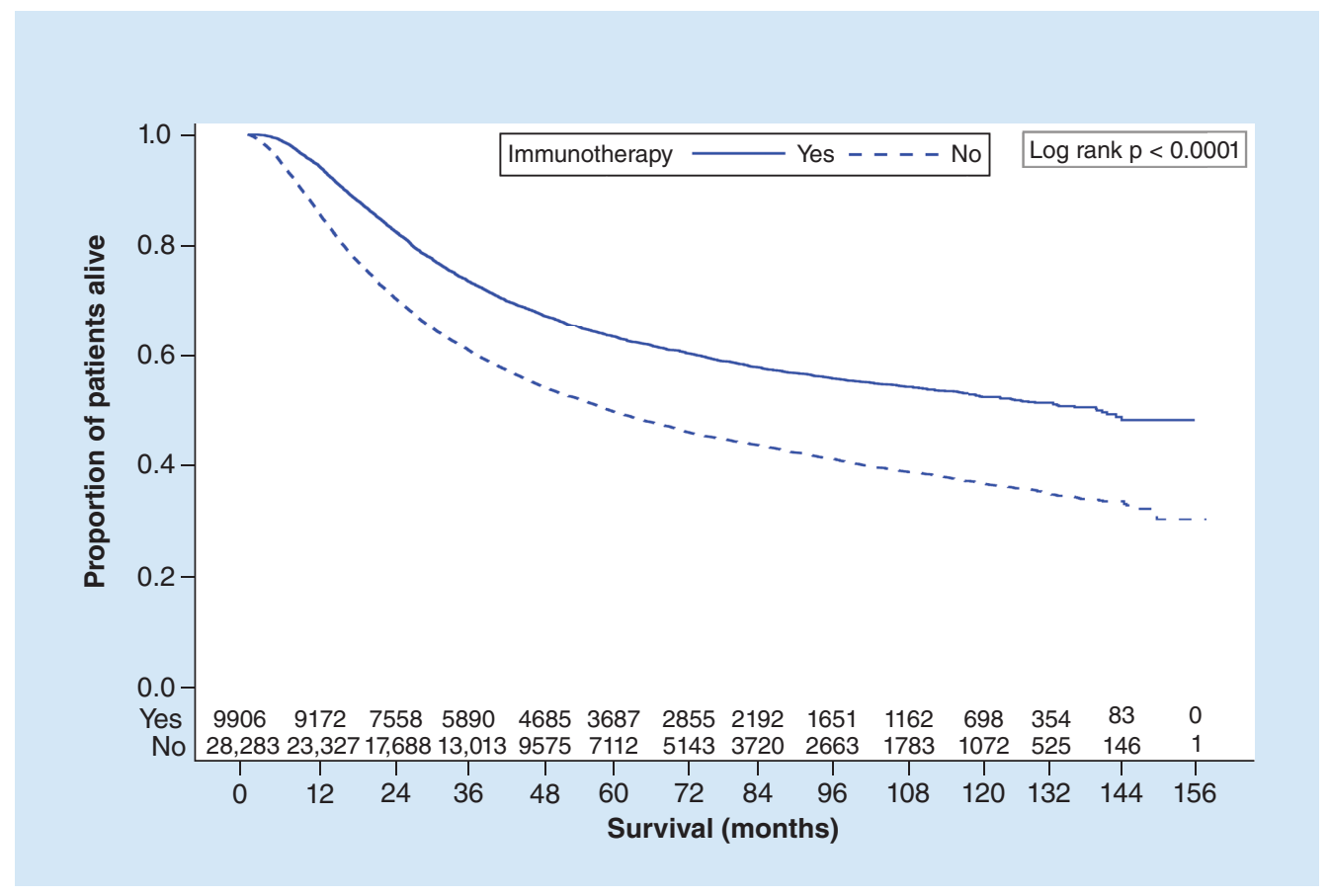

Figure 3. Kaplan-Meier overall survival plot with number at risk by immunotherapy status (yes vs no).

\begin{tabular}{|c|c|c|c|c|c|}
\hline \multirow[t]{2}{*}{ Variable } & \multirow[t]{2}{*}{ Class } & \multicolumn{2}{|c|}{$<65$ years } & \multicolumn{2}{|c|}{$\geq 65$ years } \\
\hline & & HR (95\% Cl) & p-value & HR (95\% Cl) & $p$-value \\
\hline Sex & Female & $0.68(0.65-0.71)$ & $<0.0001$ & $0.86(0.82-0.90)$ & $<0.0001$ \\
\hline \multirow[t]{2}{*}{ Race } & Caucasian & Reference & - & Reference & - \\
\hline & Other & $1.02(0.95-1.09)$ & 0.6518 & $1.00(0.93-1.08)$ & 0.9990 \\
\hline \multirow{3}{*}{ Insurance Status } & $\begin{array}{l}\text { Medicaid/other } \\
\text { government }\end{array}$ & $1.66(1.56-1.78)$ & $<0.0001$ & $1.33(1.11-1.58)$ & 0.0016 \\
\hline & Medicare & $1.68(1.55-1.81)$ & $<0.0001$ & $1.17(1.10-1.25)$ & $<0.0001$ \\
\hline & Not Insured & $1.50(1.39-1.62)$ & $<0.0001$ & $1.26(0.93-1.70)$ & 0.1396 \\
\hline Positive nodes, $\mathrm{n}$ & One node & Reference & - & Reference & - \\
\hline \multirow[t]{3}{*}{ Charlson-Deyo score } & 0 & Reference & - & Reference & - \\
\hline & 1 & $1.27(1.19-1.36)$ & $<0.0001$ & $1.19(1.13-1.26)$ & $<0.0001$ \\
\hline & 2 or 3 & $1.81(1.59-2.07)$ & $<0.0001$ & $1.60(1.46-1.76)$ & $<0.0001$ \\
\hline \multirow[t]{2}{*}{ Immunotherapy } & No & Reference & - & Reference & - \\
\hline & Yes & $0.76(0.72-0.79)$ & $<0.0001$ & $0.65(0.61-0.70)$ & $<0.0001$ \\
\hline
\end{tabular}

death (HR: 0.86; 95\% CI: 0.82-0.90) and patients with either Medicare or Medicaid/other government insurance had an increased risk of death compared with patients with private insurance (other insurance comparisons in Appendix A). Patients with more than one cancerous node and with a Charlson-Deyo score greater than zero also had a higher risk of death after taking into account the other variables in model including immunotherapy. 


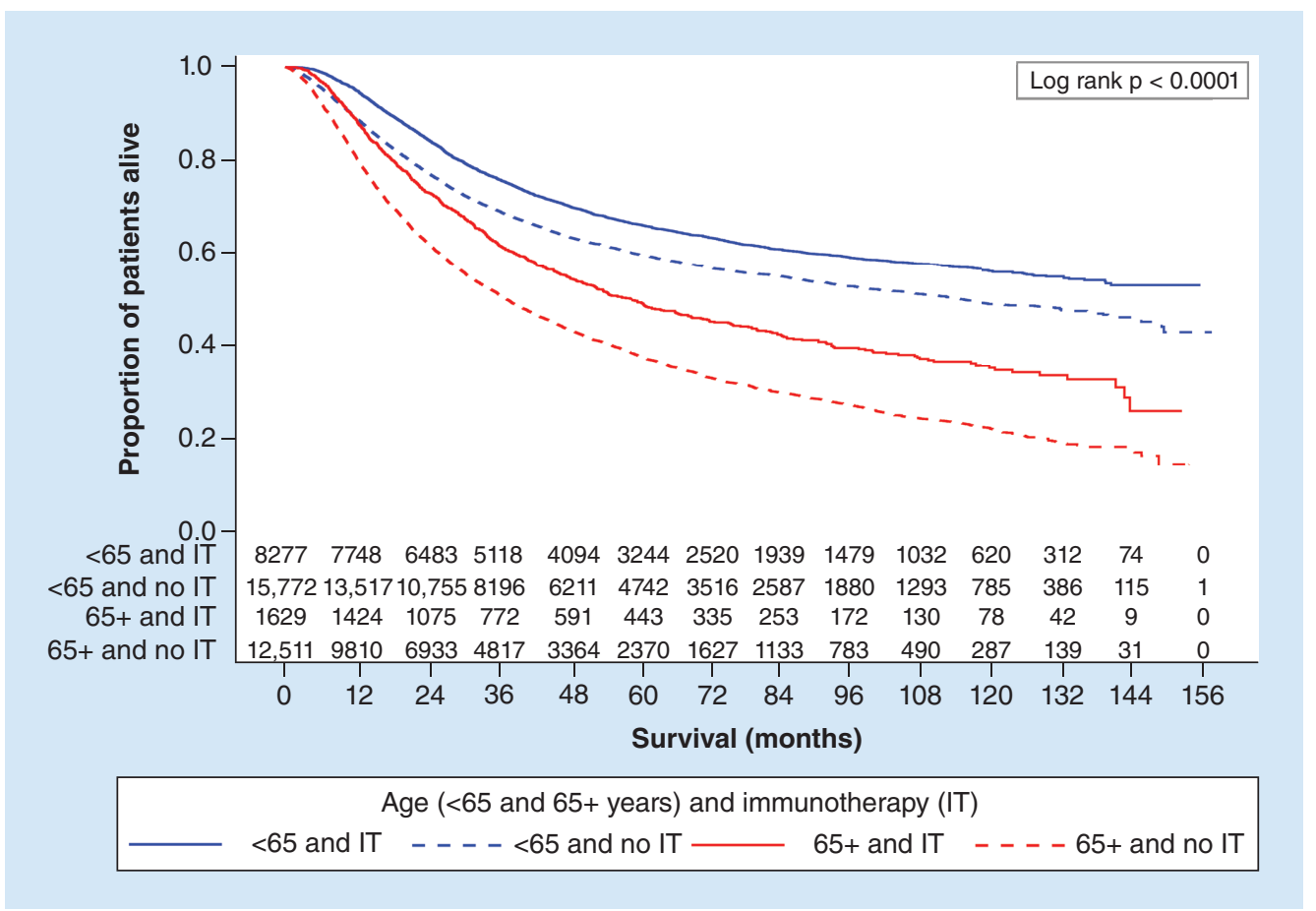

Figure 4. Kaplan-Meier overall survival plot with number at risk by age ( $<65$ vs $\geq 65$ years) and immunotherapy status (yes vs no).

IT: Immunotherapy.

\section{Discussion}

Incidence of cutaneous melanoma has increased dramatically in the recent decades, but multimodality treatment approaches for patients with advanced disease and either regional or distant metastases have also greatly expanded. Several highly effective targeted and immune checkpoint inhibitor regimens have been introduced for unresectable disease in the past 10 years and some of these therapies have now been moved into the adjuvant setting as well, based on multiple Phase III clinical trials [7,15-19] demonstrating impressive recurrence free survival. However, mature overall survival data are only reported for the earlier of these trials comparing ipilimumab to INF- $\alpha$ (ECOG 1609) or to placebo (EORTC). These two trials included just over 2600 patients cumulatively and certain subsets of patients were underrepresented. In particular, the median age of patients in all melanoma trials is typically a decade younger than the median age for melanoma diagnosis (Society for Melanoma Research Congress 2019 [UNPUBlished DATA]). Additionally, trials were largely conducted in Europe, North America and Australia, so darker-skinned racial minorities were underrepresented [20]. Our study, analyzing data from a large and unselected group of melanoma patients with node-positive disease, albeit during a time period when the most effective and least toxic adjuvant therapies were not yet available as standard of care, was conducted to fill in some of these gaps.

We were particularly interested in the question of whether older patients and racial minorities, whose melanoma and/or immune microenvironment exhibit biologic differences, would derive the same benefit as the larger population from immune-based therapies. Laboratory models and investigations have shown decreased lymphocyte motility [21] and deficient immune cell function [22,23] in the elderly. Meanwhile, emerging data on the mutational landscape of acral melanomas, which occur with relatively greater frequency in darker-skinned individuals, demonstrate approximately tenfold lower mutational burden; mutations that do occur are more typically copy number variants and fusions [10,24]. Since tumor mutational burden is highly correlated with response to immune-based therapies [25] and copy number mutations are likely to be less immunogenic than point mutations, it is theoretically possible that darker-skinned melanoma patients would have inferior outcomes when treated with this modality. An earlier report based on SEER data did, in fact, show worse survival for African Americans in comparison to Caucasians with melanoma diagnoses [8]. However, the SEER dataset was not sufficient to discern whether this outcome was due to lower response rates to therapy or to delays in receiving an unexpected diagnosis and inability to access appropriate care in a timely manner. 
Our data from the NCDB suggest that lack of receiving immunotherapy rather than insufficient benefit from the treatment itself explains survival differences in particular demographic subsets. Non-Caucasian stage III melanoma patients had a small and not quite statistically significant decrease in survival when race was considered in univariate analysis, but this difference dropped out in multivariable analysis considering factors such as payer type, which can influence access to care; there was no difference in outcome between Caucasians and non-Caucasians who received adjuvant immunotherapy. The comparison of patients $<65$ or $\geq 65$ years of age who received adjuvant immunotherapy actually showed a beneficial interaction with older age. While older patients had higher mortality overall, they had a more significant improvement in survival (HR: 0.65 vs 0.76 ) than younger patients, when treated with immunotherapy, after controlling for known risk factors including sex, race, number of positive nodes, insurance status and Charlson-Deyo score. A review of the effect of age on melanoma risk, prognosis and treatment response [11] also indicates a complex interaction between age and survival. Lower stage specific survival is reported overall and the benefit of lower incidence of positive sentinel lymph node is offset by worse outcome when a positive sentinel node is detected. However, intriguingly, data from several large single institutions [26-28] as well as pooled data from high volume melanoma treatment centers [29] show superior survival advantage in older patients treated with various ICI regimens for metastatic disease, results that comport with our findings from analysis of the NCDB.

Our study also has limitations, in that we do not have specific information regarding each individual's therapy in this large database. A similarly powered comparison comes from a meta-analysis of 17 randomized controlled trials of adjuvant INF- $\alpha$ with about 10,000 evaluated patients [30]. This analysis demonstrated a statistically significant but smaller absolute overall survival advantage than we observed but included results of lower-dose INF- $\alpha$ regimens. We presume that off protocol immunotherapy for stage III melanoma during the 2004-2015 timeframe would have been accomplished with high-dose IFN- $\alpha$, but the available information does not conclusively establish that this is the case. Furthermore, in the few years since the cut-off for this data set, adjuvant immunotherapy has evolved rapidly. High-dose INF- $\alpha$ was associated with frequent and significant adverse events including pyrexia, cytopenias, hepatotoxicity and neuropsychiatric effects that prevented its widespread acceptance as a standard of care, despite moderate efficacy. Adjuvant ICIs, primarily those directed at PD1, cause far fewer dose limiting toxicities and are more efficacious, resulting in their routine use since late 2017. It is not possible at this time to determine if the uniform benefit to underrepresented demographic groups reported here persists in this altered therapeutic landscape; that analysis will require several years of additional accrual to the NCDB. Until an updated review can be accomplished, however, the data reported here could provide a reasonable basis to offer adjuvant immunotherapy to all node-positive melanoma patients with no presumption of decreased benefit based on demographic factors.

\section{Future perspective}

Adjuvant immunotherapy for lymph node-positive melanoma has already advanced rapidly in the recent past, but further evolution in the next $5-10$ years is still likely. A clinical trial of combination ICIs in comparison to single agent nivolumab (CheckMate 915, NCT03068455) has completed accrual and preliminary survival data for the entire intent to treat population can be expected in the next 1-2 years. A proposed Southwest Oncology Group protocol (S1935 per Fall 2019 Agenda Book) anticipates evaluating a targeted therapy plus ICI combination in the adjuvant setting for patients with activating $B R A F$ mutations. Analyzing the impact of novel melanoma therapies on demographic subsets as we have reported will thus always be a moving target. However, we would project that a similar review of NCDB outcomes in 2024-2025 would reflect results of current standard of care regimens.

Supplementary data

To view the supplementary data that accompany this paper please visit the journal website at: www.futuremedicine.com/doi/sup $\mathrm{pl} / 10.2217 / \mathrm{mmt}-2020-0002$

\section{Author contributions}

A Ikeguchi conceived of the work and acquired the data; M Machiorlatti and SK Vesely analyzed the data. All authors contributed to the interpretation of the data, drafting the work and revising it critically for important intellectual content and final approval of the version to be published. All authors agree to be accountable for all aspects of the work to ensure accuracy and integrity. 
Summary points

Background

- Melanoma has increased in incidence.

- Adjuvant immunotherapy has been shown to confer survival benefit for patients with lymph node positive disease.

- Demographic groups such as racial minorities and the elderly are underrepresented in clinical trials of adjuvant treatment.

Materials and methods

- We evaluated a large database of melanoma patients treated according to existing standard of care.

- Statistical analyses were performed to compare treatment outcome of patients from underrepresented demographic groups.

Results

- Factors predicting for worse outcome in univariate analysis were male sex, age $\geq 65$ years, higher nodal involvement, increased comorbidity score, no immunotherapy, and no insurance.

- In multivariable analysis, none of the factors interacted to diminish benefit of immunotherapy.

- Increasing age showed positive interaction with benefit of immunotherapy.

Discussion

- This analysis demonstrated that demographic groups underrepresented in clinical trials of adjuvant immunotherapy for lymph node positive melanoma derive survival benefit from treatment.

Future perspective

- A similar analysis performed in the future would clarify whether newer and less toxic adjuvant immunotherapies also confer benefit across all demographic subgroups.

Financial \& competing interests disclosure

Research reported in this publication was supported in part by the National Cancer Institute Cancer Center Support Grant P30CA225520 awarded to the University of Oklahoma Stephenson Cancer Center and used the Biostatistics and Research Design Shared Resource. The content is solely the responsibility of the authors and does not necessarily represent the official views of the $\mathrm{NIH}$. The authors have no other relevant affiliations or financial involvement with any organization or entity with a financial interest in or financial conflict with the subject matter or materials discussed in the manuscript apart from those disclosed.

No writing assistance was utilized in the production of this manuscript.

\section{Open access}

This work is licensed under the Attribution-NonCommercial-NoDerivatives 4.0 Unported License. To view a copy of this license, visit http://creativecommons.org/licenses/by-nc-nd/4.0/

\section{References}

Papers of special note have been highlighted as: • of interest

1. Siegel R, Miller K, Jemal A. Cancer statistics (2018). CA Cancer J. Clin. 68(1), 7-30 (2018).

2. Surveillance, Epidemiology and End Results. Cancer Stat Facts: Melanoma of the Skin. https://seer.cancer.gov/statfacts $/ \mathrm{html} / \mathrm{melan} . \mathrm{html}$

3. Gershenwald JE, Scolyer RA, Hess KR et al. Melanoma staging: evidence-based changes in the American Joint Committee on Cancer eighth edition cancer staging manual. CA Cancer J. Clin. 67(6), 472-492 (2017).

4. Hodi FS, O'day SJ, Mcdermott DF et al. Improved survival with ipilimumab in patients with metastatic melanoma. N. Engl. J. Med. 363(8), 711-723 (2010).

5. Chapman PB, Hauschild A, Robert $\mathrm{C}$ et al. Improved survival with vemurafenib in melanoma with BRAF V600E mutation. N. Engl. J. Med. 364(26), 2507-2516 (2011).

6. Robert C, Schachter J, Long GV et al. Pembrolizumab versus ipilimumab in advanced melanoma. N. Engl. J. Med. 372(26), 2521-2532 (2015).

7. Weber J, Mandala M, Del Vecchio M et al. Adjuvant nivolumab versus ipilimumab in resected stage III or IV melanoma. N. Engl. J. Med. 377(19), 1824-1835 (2017).

8. Mahendraraj K, Sidhu K, Lau CS, Mcroy GJ, Chamberlain RS, Smith FO. Malignant melanoma in African-Americans: a population-based clinical outcomes study involving 1106 African-American patients from the surveillance, epidemiology and end result (SEER) Database (1988-2011). Medicine 96(15), e6258 (2017).

- Reports an analysis of SEER data indicating excess mortality in African-Americans diagnosed with melanoma. 
9. Moon KR, Choi YD, Kim JM et al. Genetic alterations in primary acral melanoma and acral melanocytic nevus in Korea: common mutated genes show distinct cytomorphological features. J. Invest. Dermatol. 138(4), 933-945 (2018).

10. Furney SJ, Turajlic S, Stamp G et al. The mutational burden of acral melanoma revealed by whole-genome sequencing and comparative analysis. Pigm. Cell Melanoma R. 27(5), 835-838 (2014).

- First report of mutational burden from whole genome sequencing specifically of acral melanoma.

11. Ribero S, Stucci LS, Marra E et al. Effect of age on melanoma risk, prognosis and treatment response. Acta Derm. Venereol. 98(7), 624-629 (2018).

12. American College of Surgeons. National Cancer Database. https://www.facs.org/quality-programs/cancer/ncdb

13. Deyo RA, Cherkin DC, Ciol MA. Adapting a clinical comorbidity index for use with ICD-9-CM administrative databases. J. Clin. Epidemiol. 45(6), 613-619 (1992).

14. American College of Surgeons. National Cancer Database. http://ncdbpuf.facs.org/content/charlsondeyo-comorbidity-index

15. Kirkwood JM, Strawderman MH, Ernstoff MS, Smith TJ, Borden EC, Blum RH. Interferon alfa-2b adjuvant therapy of high-risk resected cutaneous melanoma: the Eastern Cooperative Oncology Group Trial EST 1684. J. Clin. Oncol. 14(1), 7-17 (1996).

16. Eggermont AM, Suciu S, Santinami M et al. Adjuvant therapy with pegylated interferon alfa-2b versus observation alone in resected stage III melanoma: final results of EORTC 18991, a randomised Phase III trial. Lancet 372(9633), 117-126 (2008).

17. Eggermont AM, Chiarion-Sileni V, Grob J-J et al. Adjuvant ipilimumab versus placebo after complete resection of high-risk stage III melanoma (EORTC 18071): a randomised, double-blind, Phase 3 trial. Lancet Oncol. 16(5), 522-530 (2015).

18. Tarhini AA, Lee SJ, Hodi FS et al. Phase III study of adjuvant ipilimumab (3 or $10 \mathrm{mg} / \mathrm{kg}$ ) versus high-dose interferon alfa-2b for resected high-risk melanoma: North American intergroup E1609. J. Clin. Oncol. 38(6), 567-575 (2019).

- Reports on findings suggesting that the aged skin microenvironment is expected to enhance melanoma metastasis and inhibit immune cellular response.

19. Eggermont AMM, Blank CU, Mandala M et al. Adjuvant pembrolizumab versus placebo in resected stage III melanoma. N. Engl. J. Med. 378(19), 1789-1801 (2018).

20. U.S. Department of Health and Human Services: Food and Drug Administration. FDA report: Collection, analysis and availability of demographic subgroup data for FDA-approved medical products (2013). https://www.fda.gov/media/86561/download

21. Kaur A, Ecker BL, Douglass SM et al. Remodeling of the collagen matrix in aging skin promotes melanoma metastasis and affects immune cell motility. Cancer Discov. 9(1), 64-81 (2019).

- Provides a comprehensive update on the mutational landscape of acral melanoma.

22. Jackaman C, Radley-Crabb HG, Soffe Z, Shavlakadze T, Grounds MD, Nelson DJ. Targeting macrophages rescues age-related immune deficiencies in C57 BL/6J geriatric mice. Aging Cell 12(3), 345-357 (2013).

- Provides an early report of correlation between overall mutational burden and response to immune checkpoint inhibitor therapy.

23. Lages CS, Lewkowich I, Sproles A, Wills-Karp M, Chougnet C. Partial restoration of T-cell function in aged mice by in vitro blockade of the PD-1/PD-L1 pathway. Aging Cell 9(5), 785-798 (2010).

24. Hayward NK, Wilmott JS, Waddell N et al. Whole-genome landscapes of major melanoma subtypes. Nature 545(7653), 175-180 (2017).

25. Snyder A, Makarov V, Merghoub T et al. Genetic basis for clinical response to CTLA-4 blockade in melanoma. N. Engl. J. Med. 371(23), 2189-2199 (2014).

26. Elias R, Karantanos T, Sira E, Hartshorn KL. Immunotherapy comes of age: immune aging \& checkpoint inhibitors. J. Geriatr. Oncol. 8(3), 229-235 (2017).

- Reports the largest analysis of immune checkpoint inhibitor therapeutic outcomes for older (>age 60) patients with unresectable melanoma.

27. Betof AS, Nipp RD, Giobbie-Hurder A et al. Impact of age on outcomes with immunotherapy for patients with melanoma. Oncologist 22(8), 963 (2017).

28. Friedman CF, Horvat TZ, Minehart J et al. Efficacy and safety of checkpoint blockade for treatment of advanced melanoma (mel) in patients (pts) age 80 and older (80+). J. Clin. Oncol. 34, 10009-10009 (2016).

29. Kugel CH, Douglass SM, Webster MR et al. Age correlates with response to anti-PD1, reflecting age-related differences in intratumoral effector and regulatory T-cell populations. Clin. Cancer Res. 24(21), 5347-5356 (2018).

30. Mocellin S, Lens MB, Pasquali S, Pilati P, Sileni VC. Interferon alpha for the adjuvant treatment of cutaneous melanoma. Cochrane Database Syst. Rev. 6, CD008955 (2013). 